\title{
Paraoxonase activity in sera of patients with non-alcoholic fatty liver disease
}

\author{
Fedelesova $\mathrm{M}^{1}$, Kupcova $\mathrm{V}^{1}$, Luha $\mathrm{J}^{3}$, Turecky $\mathrm{L}^{2}$ \\ Institute of Medical Chemistry, Biochemistry and Clinical Biochemistry, Medical Faculty of Comenius \\ University, Bratislava, Slovakia. ladislav.turecky@fmed.uniba.sk
}

\section{ABSTRACT}

The results of our study showed significantly decreased levels of PON1 in patients with chronic liver diseases (controls $185 \pm 14 \mathrm{U} / \mathrm{I}, \mathrm{NAFLD} 160 \pm 15 \mathrm{U} / \mathrm{l}$, chronic hepatitis $99 \pm 18 \mathrm{U} / \mathrm{l}$, cirrhosis $52 \pm 11 \mathrm{U} / \mathrm{l}$ ). There were significant correlations of PON activities with standard liver function tests (Tab. 1, Ref. 5). Text in PDF www.elis.sk. KEY WORDS: paraoxonase, fatty liver disease, oxidative stress, NAFLD.

Non-alcoholic fatty liver disease (NAFLD) affects 10-24\% of the general population and is usually associated with insulin resistance, central obesity and hyperlipidaemia. Female gender, obesity, hyperlipidaemia and diabetes mellitus are well-known risk factors of NAFLD. The pathogenesis of NAFLD remains unclear, although two mechanisms are implicated in the development of this disorder: increased oxidative stress and lipid peroxidation associated with an increased fat deposition in the liver $(1,2)$.

Paraoxonases are a family of three enzymes termed PON1, PON2 and PON3. The best known enzyme among the paraoxonases is PON1. It is an ester hydrolase that catalyses the hydrolysis of some xenobiotics. The liver plays a key role in the synthesis of serum PON1. PON1 in serum is entirely complexed to HDL, it hydrolyses lipid peroxides and plays a role in the antioxidant system and in the regulation of oxidative stress. The aim of our study was to investigate the relationship between the activity of serum PON1 and liver function in patients with NAFLD and other chronic liver diseases.

\section{Patients and methods}

The study population consisted of 65 patients with chronic hepatopathies (liver cirrhosis, hepatitis, NAFLD) and 19 healthy controls. Determination of PON1 activity was analysed with paraoxon as a substrate. The levels of prealbumin and albumin were determined by electroimmunoassay.

\footnotetext{
${ }^{13 r d}$ Department of Internal Medicine, University Hospital, Medical faculty, Comenius University, Bratislava, Slovakia, ${ }^{2}$ Institute of Medical Chemistry, Biochemistry and Clinical Biochemistry, Medical Faculty, Comenius University, Bratislava, Slovakia, and ${ }^{3}$ Institute of Medical Biology and Clinical Genetics, Medical Faculty, Comenius University, Bratislava, Slovakia

Address for correspondence: $\mathrm{L}$. Turecky, MD, PhD, Institute of Medical Chemistry, Biochemistry and Clinical Biochemistry, Medical School, Comenius University, Sasinkova 2, SK-811 08 Bratislava, Slovakia.

Acknowledgement: This work is supported by the Scientific Grant Agency of the Ministry of Education of Slovak Republic and the Slovak Academy of Sciences VEGA 1/0808/15.
}

Tab. 1. The significant correlations of PON activities with standard liver function tests.

\begin{tabular}{lccc}
\hline Group & $\begin{array}{c}\text { PON1 } \\
\mathrm{U} / \mathrm{l}\end{array}$ & $\begin{array}{c}\text { Prealbumin } \\
\mathrm{mg} / \mathrm{l}\end{array}$ & $\begin{array}{c}\text { Albumin } \\
\mathrm{g} / \mathrm{l}\end{array}$ \\
\hline Controls $\mathrm{n}=19$ & $185 \pm 14$ & $325 \pm 21$ & $41 \pm 5$ \\
NAFLD $\mathrm{n}=19$ & $160 \pm 15^{*}$ & $301 \pm 29 \mathrm{~ns}$ & $38 \pm 5 \mathrm{~ns}$ \\
Chronic hepatitis $\mathrm{n}=21$ & $99 \pm 18^{* * *}$ & $250 \pm 28^{* * *}$ & $31 \pm 7^{* *}$ \\
Liver cirrhosis $\mathrm{n}=25$ & $52 \pm 11^{* * *}$ & $108 \pm 22^{* * *}$ & $24 \pm 5$ \\
\hline
\end{tabular}

Results are given as the mean \pm SEM, significance of the group compared to controls, ${ }^{*} \mathrm{p}<0.05,{ }^{* *} \mathrm{p}<0.01,{ }^{* * *} \mathrm{p}<0.001$, ns not significant

\section{Results}

The results of our study showed significantly decreased levels of PON1 in patients with chronic liver diseases (controls $185 \pm 14$ U/l, NAFLD $160 \pm 15 \mathrm{U} / \mathrm{l}$, chronic hepatitis $99 \pm 18 \mathrm{U} / \mathrm{l}$, cirrhosis $52 \pm 11 \mathrm{U} / \mathrm{l})$. There were significant correlations of PON activities with standard liver function tests (Tab. 1).

\section{Discussion}

The significant decrease of PON1 activity in chronic liver diseases was related to the degree of hepatic dysfunction. Our finding of decreased activities of PON1 in sera of patients with NAFLD is in agreement with the findings of Atamer et al (1) and Wang et al (2). The decreased PON1 activities in other chronic hepatopathies are also in agreement with data from human and experimental studies $(3,4)$. The physiological role played by PON1 in the liver is unknown, although preliminary observations suggest its role in hepatic protection against oxidative stress. The decreased activity of PON1 as an antioxidant could play a role in the defective antioxidant defence and participate in the increase of oxidative stress. On the other hand, decreased activity of PON1 in patients with chronic liver diseases could be the result of damage of hepatocytes and reduced volume of functional liver parenchyma. This suggestion is supported by a good correlation between PON1 activities and 


\section{9-720}

levels of prealbumin and albumin, which are accepted as parameters of liver function. The hypothesis that the differences between PON1 activities in controls and patients with hepatopathies are the consequence of differences in genotype frequency distribution in patients and controls was discarded by Ferré et al. (5), who found in their study that genotype distributions were not significantly different between the patients with hepatopathies and the controls.

\section{References}

1. Atamer A, Bilici A, Yenice N, Selek S, Ilhan N, Atamer Y. The importance of paraoxonase 1 activity, nitric oxide and lipid peroxidation in hepatosteatosis. J Int Med Res 2008; 36: 771-776.

2. Wang B, Yang RN, Zhu YR et al. Involvement of xanthine oxidase and paraoxonase 1 in the process of oxidative stress in nonalcoholic fatty liver disease. Mol Med Rep 2017; 15 (1): 387-395.
3. Marsillach J, Aragones G, Beltrán R et al. The measurement of the lactonase activity of paraoxonase- 1 in the clinical evaluation of patients with chronic liver impairment. Clin Biochem 2009; 42 (1-2): 91-98.

4. Marsillach J, Camps J, Ferré $\mathbf{N}$ et al. Paraoxonase-1 is related to inflammation, fibrosis and PPAR delta in experimental liver disease. BMC Gastroenterol 2009; 9: 1-13.

5. Ferré N, Camps J, Prats E, Vitella E, Paul A, Figuera L, Joven J. Serum paraoxonase activity: A new additional test for the improved evaluation of chronic liver damage. Clin Chem 2002; 48 (2): 261-268.

Received July 27, 2017. Accepted August 29, 2017. 